\title{
Reacciones Adversas a Medicamentos como Motivo de Consulta en un Servicio de Atención Prioritaria
}

\author{
Adverse drug reactions as the reason for visiting an \\ emergency department
}

\author{
Carlos A. Calderón-Ospinaํㅗ y José G. Orozco-Díaz ${ }^{2}$ \\ 1 Unidad de Farmacología. Departamento de Ciencias Básicas. Facultad de Medicina, Universidad \\ del Rosario. Bogotá, Colombia. carlos.calderon68@urosario.edu.co \\ 2 Unidad de Farmacología. Departamento de Ciencias Fisiológicas. Facultad de Medicina, Universidad \\ Nacional de Colombia. Bogotá. jgorozcod@unal.edu.co
}

Recibido 26 Julio 2007/Enviado para Modificación 16 Marzo 2008/Aceptado 19 Abril 2008

\section{RESUMEN}

Objetivo El objetivo del presente estudio consistió en determinar la prevalencia de las reacciones adversas a medicamentos (RAM) como motivo de consulta a un servici-o de atención prioritaria.

Métodos El estudio se realizó en el servicio de atención prioritaria del Centro de Atención Ambulatoria Central Luis de la ESE Luis Carlos Galán Sarmiento en Bo-gotá, del 20 de septiembre al 2 de octubre de 2004. Las RAM fueron identificadas por un evaluador, quien revisó todas las historias clínicas y cada vez que los médi-cos señalaron la sospecha de una RAM. Los pacientes fueron contactados. La eva-luación de la causalidad, prevenibilidad, y mecanismo de generación fue realizada por dos evaluadores.

Resultados La prevalencia de RAM fue de 45 RAM en 1395 consultas, en dos semanas $(2,8 \%$ de las consultas). El reporte espontáneo modificado permitió de-tectar el $26,7 \%$ de las RAM identificadas por búsqueda activa. Del total de las RAM identificadas el 73,3 \% se presentó en mujeres, 64,9 \% en mayores de 60 años, $48,9 \%$ fueron no prevenibles, $22,2 \%$ prevenibles y $28,9 \%$ inclasificables.

Conclusiones Las RAM representan una proporción significativa de la consulta ambulatoria y el sistema de reporte espontáneo modificado detectó un gran porcen-taje de RAM. La distribución y tipo de RAM fueron similares a las descritas en otras series. Aproximadamente 2 de cada 10 RAM pudieron haberse prevenido.

Palabras Clave: Farmacoepidemiología, utilización de medicamentos (fuente: DeCS, BIREME).

\section{ABSTRACT}

Objective Determining the prevalence of adverse drug reactions (ADR) as the reason for visiting an emergency department. 
Methods The study took place at the Luis Carlos Galán Sarmiento ESE's Emergency Department's Central Outpatient Centre in Bogotá. It was carried out from September $20^{\text {th }}$ to October $2^{\text {nd }} 2004$. ADR were identified by an evaluator who reviewed all clinical histories, noting when physicians had indicated that an ADR had occurred. The patients were contacted. Two people evaluated causality, the degree of preventability and the mechanism producing the ADR.

Results 45 ADR were recorded in 1,395 visits made during a two-week period (2.8\% of the consultations). Modified spontaneous report detected $26.7 \%$ ADR identified by active search. $73.3 \%$ of all identified ADR occurred in females, $64.9 \%$ in people older than $60.48 .9 \%$ were non-preventable, $22.2 \%$ preventable and $28.9 \%$ were unclassifiable.

Conclusions ADR represent a significant proportion of the reasons for outpatient consultation; the modified spontaneous report system detected a large percentage of ADR. There were no statistically significant differences between gender and ADR frequency. ADR occurred more frequently in elderly patients. Around 2 out of each 10 ADR could have been prevented.

Key Words: Pharmacoepidemiology, drug utilization (source: MeSH, NLM).

L as reacciones adversas a medicamentos (RAM) representan un problema de salud pública importante $(1,2)$. Una RAM se define como "Una respuesta a un fármaco que es nociva e involuntaria, y que ocurre a las dosis normalmente usadas en el hombre para profilaxis, diagnóstico o terapia de la enfermedad, o para modificación de la función fisiológica" (3).

La prevalencia de RAM en población ambulatoria en Colombia no se conoce con exactitud, aunque un estudio nacional señala que la misma podría estar alrededor del 6,4\% (4). En la literatura internacional se encuentra que la misma puede oscilar entre el 0,96 y el 10,6 \% (5-10). Se ha estimado que hasta el $28 \%$ de las RAM son prevenibles (11). Dos estudios dirigidos a encontrar el porcentaje de RAM prevenibles en población ambulatoria encontraron que este era del 20 y del 27,6 \% respectivamente $(12,13)$.

El subreporte de RAM es uno de los problemas más importantes que afronta la farmacovigilancia. Por ejemplo, en un estudio inglés dirigido a encontrar el subreporte de RAM de fármacos recién introducidos al mercado, se encontró que los médicos solo reportaron el $9 \%$ de los eventos adversos que ocurrieron (14).

El objetivo general del presente estudio consistió en determinar la prevalencia de RAM como motivo de consulta a un servicio de atención prioritaria de una institución prestadora de salud de primer nivel en Bogotá. Como objetivos secun- 
darios se contemplaron: determinar las diferencias de detección de las RAM con el sistema de reporte espontáneo modificado comparado con la búsqueda activa por los investigadores, determinar la distribución por edad y sexo de los pacientes que presentaron una RAM como motivo de consulta a un servicio de atención prioritaria, determinar la relación de causalidad entre el consumo del medicamento y la aparición de la RAM en cada caso y su distribución en la población estudiada, determinar la distribución de frecuencias según seriedad de las RAM, determinar la proporción de RAM prevenibles y determinar la distribución de las RAM según el mecanismo de generación.

\section{MÉTODOS}

Se realizó un estudio descriptivo de corte transversal. Se revisaron las historias clínicas y se realizaron entrevistas telefónicas a pacientes, sin que se llevara a cabo ninguna intervención o modificación de los tratamientos recibidos por los pacientes que participaron en el estudio. Se siguieron y respetaron las normas establecidas para la investigación en humanos, definidas en la Declaración de Helsinki y en la Resolución 8430 de 1993 del Ministerio de Salud. La información a la que se tuvo acceso fue manejada con carácter de confidencialidad y la presentación de los resultados fue manejada por conglomerados, de manera anónima, para proteger la identidad de cada uno de los individuos participantes. El protocolo fue llevado a cabo previa autorización del Instituto Nacional de Vigilancia de Medicamentos y Alimentos-INVIMA y de las directivas y el Comité de Ética del centro asistencial, y a cada paciente que fue entrevistado se le pidió su consentimiento para participar en el estudio. El estudio fue llevado a cabo en el servicio de atención prioritaria del Centro de Atención Ambulatoria (CAA) Central de la Empresa Social del Estado Luis Carlos Galán Sarmiento durante los días comprendidos entre el 20 de septiembre y el 2 de octubre de 2004.

\section{Materiales}

El cálculo del tamaño muestral fue realizado de acuerdo a los resultados obtenidos en la prueba piloto utilizando STAT CALC de EPI INFO, versión 2002, revisión 2.

De acuerdo a los objetivos del estudio se diseñó un formato de recolección de información. Junto con el formato, fue diseñada una base de datos en Microsoft Access ${ }^{\circledR}$ que contó con los mismos campos del formato de recolección de información. Posteriormente, dicha base de datos fue analizada a través del programa Microsoft Excel®, versión 2003. Los cálculos de las proporciones, los 
intervalos de confianza y las diferencias entre proporciones fue realizada con el programa EPITABLE® de EPI INFO ${ }^{\circledR}$. Para la codificación en la base de datos de las RAM, los medicamentos y los antecedentes de los pacientes se utilizaron la clasificación WHO-ART de la Organización Mundial de la Salud (OMS), la clasificación ATC de medicamentos y la clasificación internacional de enfermedades CIE 10, respectivamente.

\section{Procedimientos}

Cinco médicos participaron en el estudio y notificaron la existencia de una RAM colocando una marca en las historias clínicas cada vez que tenían la más mínima sospecha que en el cuadro clínico del paciente el medicamento podía ser un factor contribuyente. A este reporte se le llamó espontáneo modificado, por la simplicidad de este sistema de notificación.

En paralelo, un investigador evaluó el total de historias clínicas, en el método conocido como búsqueda intensiva, diligenciando un formato por cada RAM. La información faltante se completó a través de entrevista telefónica con el paciente.

A cada caso de sospecha de reacción adversa se le realizó la evaluación de causalidad de acuerdo al algoritmo de la OMS. La prevenibilidad fue evaluada empleando los criterios de Schumock y Thornton modificados (15), y el mecanismo de generación de acuerdo a la clasificación de Rawlins y Thompson modificada (16).

Una vez completados los formatos de recolección de información se diligenció la base de datos, la cual fue posteriormente analizada.

\section{RESULTADOS}

Las características basales de la población consultante se presentan en la Tabla 1.

Tabla 1. Características generales de la población estudiada

\begin{tabular}{crr}
\hline \multirow{2}{*}{ Características } & \multicolumn{2}{c}{ Pacientes } \\
\cline { 2 - 3 } & No. & \multicolumn{1}{c}{$\%$} \\
\hline Hombres & 517 & 37,1 \\
Mujeres & 878 & 63,9 \\
Edades (Años) & & \\
$0-10$ & 65 & 4,7 \\
$11-20$ & 100 & 7,2 \\
$21-30$ & 107 & 7,7 \\
$31-40$ & 182 & 13,1 \\
$41-50$ & 260 & 18,7 \\
$51-60$ & 276 & 19,8 \\
$61-70$ & 221 & 15,8 \\
$71-80$ & 138 & 9,9 \\
$81-90$ & 44 & 3,2 \\
$91-100$ & 1 & 0,1 \\
\hline
\end{tabular}


La prevalencia encontrada fue de 45 RAM (39 pacientes) en 1395 consultas, durante un periodo de dos semanas, es decir, el 2,8 \% de las consultas (IC $95 \%$ 2,02-3,76) al servicio de atención prioritaria se debieron a una RAM.

La distribución de las RAM de acuerdo a los objetivos secundarios se presenta en la Tabla 2. No se tuvo el dato de la edad para dos pacientes. Se presentaron dos casos de RAM serias.

Tabla 2. Distribución de las RAM encontradas

\begin{tabular}{|c|c|c|}
\hline \multirow{2}{*}{ Características } & \multicolumn{2}{|c|}{ Pacientes } \\
\hline & No. & $\%$ \\
\hline \multicolumn{3}{|l|}{ Sistema de búsqueda } \\
\hline Búsqueda activa & 45 & 100,0 \\
\hline Reporte espontáneo modificado & 12 & 26,7 \\
\hline \multicolumn{3}{|l|}{ Género } \\
\hline Hombres & 12 & 26,7 \\
\hline Mujeres & 33 & 73,3 \\
\hline \multicolumn{3}{|l|}{ Edad } \\
\hline$<60$ años & 15 & 35,1 \\
\hline$\geq 60$ años & 28 & 64,9 \\
\hline \multicolumn{3}{|l|}{ Causalidad } \\
\hline Probables & 9 & 20 \\
\hline Posibles & 34 & 75,6 \\
\hline Condicionales/No clasificadas & 1 & 2,2 \\
\hline Improbables & 1 & 2,2 \\
\hline \multicolumn{3}{|l|}{ Seriedad } \\
\hline Serias & 2 & 4,4 \\
\hline No serias & 43 & 95,6 \\
\hline \multicolumn{3}{|l|}{ Prevenibilidad } \\
\hline Prevenibles & 10 & 22,2 \\
\hline No prevenibles & 22 & 48,9 \\
\hline Inclasificables & 13 & 28,9 \\
\hline \multicolumn{3}{|l|}{ Mecanismo de generación } \\
\hline A & 20 & 44,4 \\
\hline $\mathrm{B}$ & 14 & 31,1 \\
\hline$x$ & 11 & 24,5 \\
\hline
\end{tabular}

\section{DISCUSIÓN}

La prevalencia de RAM encontrada en el presente estudio (2,8 \%) no es despreciable y sirve como un estimado de la prevalencia de RAM en población ambulatoria en el medio urbano colombiano.

Una de las limitaciones más importantes del presente estudio consiste en que la identificación de las RAM dependió exclusivamente del criterio de un único evaluador, Sin embargo, la prevalencia encontrada en el presente estudio está de acuerdo con otros estudios.

La detección del 26,7 \% de las RAM con el sistema de reporte espontáneo modificado representa un dato interesante, dada la elevada proporción de 
subregistro con el reporte voluntario tradicional, lo que permite proponerlo como una alternativa novedosa para el desarrollo de programas de farmacovigilancia al interior de las instituciones asistenciales.

Sobre la relación de causalidad de los diferentes fármacos en la generación de las RAM se encuentra que hay un franco predominio de las catalogadas como posibles seguidas por aquellas catalogadas como probables. Es claro que es muy difícil caracterizar una RAM como definitiva, puesto que la gran mayoría de algoritmos exigen la reaparición de la reacción adversa tras la reexposición, lo cual ocurre de manera infrecuente en la práctica clínica habitual.

La gran mayoría de las RAM correspondieron a reacciones no serias mientras un pequeño porcentaje correspondió a reacciones serias. Estas cifras están de acuerdo con el tipo de institución en que fue llevado a cabo el estudio. El 22,2 $\%$ de las RAM fueron consideradas como prevenibles. Quedó de manifiesto en el presente estudio que las causas más frecuentes de RAM prevenibles fueron la falta de monitoreo terapéutico de fármacos, así como la falta de cumplimiento de la terapia; problemas cuya corrección redundaría en disminución de la frecuencia de presentación y/o severidad de las RAM.

De acuerdo al mecanismo de generación los resultados están de acuerdo con un principio farmacológico elemental que consiste en que si las reacciones tipo $\mathrm{A}$ dependen de las propiedades farmacológicas del producto, deben producirse con una frecuencia mucho más alta. Se considera de gran valor la inclusión de la categoría X para caracterizar algunas RAM que no se pueden clasificar satisfactoriamente dentro de las categorías A y B.

Aunque no fue explorado en el presente estudio, se plantea la necesidad de desarrollar métodos tendientes a evaluar el impacto de este tipo de programas en la calidad de la prescripción, pues debe ser siempre este el ánimo fundamental de cualquier programa de farmacovigilancia.

En la exploración de asociaciones no se encontró diferencias en la distribución por género $(p=0,14)$, hubo mayor frecuencia en mayores de 60 años $(p=0,0000)$, concordante con lo reportado en otros estudios (7-9).

En conclusión, las RAM representan una proporción significativa de consultas en población ambulatoria en nuestro medio. El sistema de reporte espontáneo modificado detectó un porcentaje significativo de RAM, por lo que se propone como una alternativa válida tendiente a aumentar el número de reportes en ins- 
tituciones asistenciales. Sin embargo, se requieren estudios adicionales para evaluar su viabilidad a largo plazo y el mantenimiento en la tasa de notificación. Finalmente se plantea la necesidad de desarrollar métodos tendientes a evaluar el impacto de este tipo de programas en la calidad de la prescripción a través del desarrollo de estudios de utilización de medicamentos *

\section{REFERENCIAS}

1. World Health Organization-The Uppsala Monitoring Centre. The Importance of Pharmacovigilance; 2002.

2. Lazarou J, Pomeranz BH, Corey PN. Incidence of adverse drug reactions in hospitalized patients: a meta-analysis of prospective studies. JAMA. 1998; 279: 1200-1205.

3. World Health Organization-The Uppsala Monitoring Centre. Viewpoint, Part 1; 2003.

4. Bustamante C, Calderón C, Castillo I, Cifuentes P, Ramírez J. Reacciones adversas a medicamentos como motivo de ingreso al servicio de urgencias de la Clínica Universitaria Teletón. Cuadernos de Medicina en Investigación y Salud. 2007; 1: 33 - 41.

5. Orozco J, Holguín E. Reacciones adversas a medicamento: Una experiencia. Memorias encuentro de experiencias nacionales e internacionales de farmacovigilancia y tecnovigilancia. INVIMA; Noviembre 2004.

6. Güemes M, Sanz E, García M. Reacciones adversas y problemas relacionados con medicamentos en un servicio de urgencia. Revista Española de Salud Pública. 1999; 73: 511-518.

7. Muñoz M, Ayani I, Rodríguez-Sasiain M, Gutiérrez G, Aguirre C. Monitorización en un servicio de urgencias de reacciones adversas causadas por medicamentos en niños y adultos. Medicina Clínica. 1998; 111: 92 - 98.

8. Raschetti R, Morgutti M, Menniti-Ippolito F, Belisari A, Rossignoli A, Longhini P, La Guidara C. Suspected adverse drug events requiring emergency department visits or hospital admissions. European Journal of Clinical Pharmacology. 1999; 54: 959-963.

9. Michele C, Dankoff J, Colacone A, Afilalo M. Polypharmacy, adverse drug-related events, and potencial adverse drug interactions in elderly patients presenting to an emergency department. Annals of Emergency Medicine. 2001; 38: 666-671.

10. Bednall R, McRobbie D, Hicks A. Identification of medication-related attendances at an A and E department. Journal of Clinical Pharmacy and Therapeutics. 2002; 28: 41-45.

11. Nieremberg D, Melmon K. Introduction to clinical pharmacology and rational therapeutics. In: Clinical Pharmacology. 10th edition. New York; 2006.

12. Gandhi T, Weingart S, Borus J, Seger A, Peterson J, Burdick E, Seger D, Shu K, Federico F, Leape L, Bates D. Adverse drug events in ambulatory care. The New England Journal of Medicine. 2003; 348: 1556-1564.

13. Gurwitz J, Field T, Harrold L, Rothschild J, Debellis K, Seger A, Cadoret C, Fish L, Garber L, Kelleher M, Bates D. Incidence and preventability of adverse drug events among older persons in the ambulatory setting. JAMA. 2003; 289: 1107-1116.

14. Martin R, Kapoor K, Wilton L, Mann R. Underreporting of suspected adverse drug reactions to newly marketed ("black triangle") drugs in general practice: observational study. BMJ. 1998; 317: 119-120.

15.Lagnaoui R, Moore N, Fach J, Longy-Boursier M, Bégaud B. Adverse drug reactions in a department of systemic diseases-oriented internal medicine: prevalence, incidence, direct costs and avoidavility. European Journal of Clinical Pharmacology. 2000; 55: 18 -186.

16. Hunziker T, Bruppacher R, Kuenzi U, Maibach R, Braunschweig S, Halter F, Hoigné R. Classification of ADRs: a proposal for harmonization and differentiation based on the experience of the Comprehensive Hospital Drug Monitoring Bern/St. Gallen, 1974-1993. Pharmacoepidemiology and Drug Safety. 2002; 11: 159-163. 